\title{
REMARKS ON COMMUTING INVOLUTIONS
}

\section{LAWRENCE CONLON}

In [3, p. 293] R. Hermann poses the following problem (without the restriction that $G$ be simple).

(A) Given $s_{1}$ and $s_{2}$ nontrivial involutive automorphisms of a compact simple Lie group $G$, find $x \in G$ such that $\operatorname{Ad}(x) s_{1} \operatorname{Ad}(x)^{-1}$ commutes with $s_{2}$.

We wish to discuss the existence of solutions for (A). Without real loss of generality we assume $G$ simply connected. The respective fixed point groups of $s_{1}$ and $s_{2}$ are closed connected subgroups $K_{1}$ and $K_{2}$ of $G$, and $K_{1}$ acts from the left on $G / K_{2}$. In both [1] and [3] it is shown that there is a flat geodesically imbedded torus $T \subset G / K_{2}$ which meets orthogonally every $K_{1}$-orbit. Furthermore, if the decompositions of the Lie algebra $\mathfrak{g}$ of $G$ into +1 and -1 eigenspaces are given respectively by

$$
\begin{aligned}
& \mathfrak{g}=\mathfrak{l}_{1} \oplus \mathfrak{m}_{1} \\
& \mathfrak{g}=\mathfrak{f}_{2} \oplus \mathfrak{m}_{2}
\end{aligned}
$$

then $T$ has as universal covering a maximal abelian subalgebra $t$ of $\mathfrak{m}_{1} \cap \mathfrak{m}_{2}$. Indeed, $T$ may be so chosen that, under the standard imbedding $G / K_{2} \subset G$ (given by $z K_{2} \rightarrow s_{2}(z) z^{-1}$ ), it becomes identified with $\exp (\mathrm{t})$. A complete description of the singular set in $T$ is given in [1] by a finite system $\mathfrak{A}$ (called an "affine root system") of affine functionals defined on $t$.

(B) ThEOREM. (A) has a solution $x \in G$ if and only if some translation in $\mathrm{t}$ carries $\mathfrak{A}$ to a system $\mathfrak{A}^{\prime}$ such that $\omega(0)=0$ or $\frac{1}{2}$, for all $\omega \in \mathfrak{A}^{\prime}$.

Proof. By [1, pp. 233-234] translations in $t$ correspond to replacing $K_{1}$ by $\operatorname{Ad}(x) K_{1}$ for suitable $x \in T$, hence to replacing $s_{1}$ by $\operatorname{Ad}(x) s_{1} \operatorname{Ad}(x)^{-1}=s_{1}^{\prime}$. If such a translation produces a system $\mathfrak{A}^{\prime}$ in which $\omega(0)=0$ or $\frac{1}{2}$, for every $\omega \in \mathfrak{A}^{\prime}$, then by [1, Proposition S-3], $s_{1}^{\prime} s_{2}$ is an involution. It follows that $s_{1}^{\prime} s_{2}=s_{2} s_{1}^{\prime}$; hence $x \in T \subset G$ solves (A).

For the converse, let $x \in G$ be such that $\operatorname{Ad}(x) s_{1} \operatorname{Ad}(x)^{-1}$ commutes with $s_{2}$. We must show that $x$ can be chosen as an element of $T$, in which case [1] will show that the affine root system translates to a system with all constant terms 0 or $\frac{1}{2}$. From Proposition 1.4 of [1] we easily see that $G=K_{2} T K_{1}$. Write $x=x_{2} y x_{1}$ for suitable $y \in T$,

Received by the editors October 29, 1968. 
$x_{i} \in K_{i}, i=1,2$. Then

$$
\operatorname{Ad}(x) s_{1} \operatorname{Ad}(x)^{-1}=\operatorname{Ad}\left(x_{2} y\right) s_{1} \operatorname{Ad}\left(x_{2} y\right)^{-1}
$$

and this commutes with $s_{2}$. Therefore, $\operatorname{Ad}(y) s_{1} \operatorname{Ad}(y)^{-1}$ commutes with $\operatorname{Ad}\left(x_{2}\right)^{-1} s_{2} \operatorname{Ad}\left(x_{2}\right)=s_{2}$. q.e.d.

The classification [2] has shown via (B) that (A) fails to have a solution in the cases equivalent to the following.

$-\frac{K_{1}}{\mathrm{SU}(2 q)} \mid$\begin{tabular}{c|c}
$K_{2}$ \\
\hline $\mathrm{Sp}(q)$ & $U(2 q-1)$
\end{tabular}

(2)

(6)

\begin{tabular}{|c|c|c|}
\hline $\begin{array}{l}\mathrm{SU}(2 r+2 q) \\
(q>r+1)\end{array}$ & $\operatorname{Sp}(r+q)$ & $S(U(2 q-1) \times U(2 r+1))$ \\
\hline $\operatorname{Spin}(2 q)$ & $U(q)$ & $\operatorname{Spin}(2 q-1)$ \\
\hline $\begin{array}{r}\operatorname{Spin}(2 r+2 q+2) \\
(q>r)\end{array}$ & $U(r+q+1)$ & $\operatorname{Spin}(2 r+1) \times_{z_{2}} \operatorname{Spin}(2 q+1)$ \\
\hline $\operatorname{Spin}(8)$ & $\operatorname{Spin}(7)$ & $\omega(\operatorname{Spin}(7))$ \\
\hline Spin(8) & $\operatorname{Spin}(7)$ & $\omega\left(\operatorname{Spin}(3) X_{z_{2}} \operatorname{Spin}(5)\right)$ \\
\hline $\operatorname{Spin}(8)$ & $\operatorname{Spin}(3) \times_{z_{2}} \operatorname{Spin}(5)$ & $\omega\left(\operatorname{Spin}(3) \times z_{2} \operatorname{Spin}(5)\right)$ \\
\hline
\end{tabular}

Here $\omega$ is the triality automorphism of $\operatorname{Spin}(8)$ and the various subgroups are standardly imbedded. In all other cases (A) has a solution.

Hermann shows [3, Proposition 2.1] that $K_{1}$ has a totally geodesic orbit in $G / K_{2}$ if and only if (A) can be solved. Actually, as the following proposition shows, the cases in which $K_{1}$ is transitive on $G / K_{2}$ constitute technical counterexamples to Hermann's result (and were implicitly excluded in his proof).

(C) Proposition. If $K_{1}$ is transitive on $G / K_{2}$, then (A) cannot be solved. 
Proof. Suppose the action transitive. If (A) has a solution $x \in G$, then $\operatorname{Ad}(x) K_{1}$ is also transitive on $G / K_{2}$, so we may as well assume $s_{1} s_{2}=s_{2} s_{1}$. Necessarily $K_{1} K_{2}=G$; hence $\mathfrak{l}_{1}+\mathfrak{f}_{2}=g$ and $\mathfrak{m}_{1} \cap \mathfrak{m}_{2}=0 . s_{1} s_{2}$ is an involutive automorphism of $g$ with fixed point algebra $\mathfrak{l}_{1} \cap \mathfrak{f}_{2}$ and -1 eigenspace $\mathfrak{m}_{1} \oplus \mathfrak{m}_{2}$. Thus $\mathfrak{g}=\mathfrak{l}_{1} \cap \mathfrak{l}_{2} \oplus\left(\mathfrak{m}_{1} \oplus \mathfrak{m}_{2}\right)$ is the decomposition corresponding to some symmetric space. But $\operatorname{ad}_{\mathfrak{m}_{1} \oplus \mathfrak{m}_{2}}\left(\mathfrak{l}_{1} \cap \mathfrak{l}_{2}\right)$ is a reducible representation, $\mathfrak{m}_{1}$ and $\mathfrak{m}_{2}$ being invariant subspaces. By standard theory of symmetric spaces, this contradicts the fact that $\mathfrak{g}$ is simple. q.e.d.

The transitive cases in the above table are precisely (1), (3), (5), and (6) (cf. [2], [4]).

Finally, we remark that a more careful study of the affine root system $\mathfrak{A}$ permits a complete description of the totally geodesic $K_{1}$ orbits in $G / K_{2}$ (cf. [2]).

\section{REFERENCES}

1. L. Conlon, The topology of certain spaces of paths on a compact symmetric space, Trans. Amer. Math. Soc. 112 (1964), 228-248.

2. Classification of affine root systems and applications to the theory of symmetric spaces, Mimeographed Notes, Washington University, St. Louis, Missouri, 1968.

3. R. Hermann, Totally geodesic orbits of groups of isometries, Nederl. Akad. Wetensch. Proc. Ser. A 65 (1962), 291-298.

4. A. L. Oniščik, Inclusion relations between transitive compact transformation groups, Trudy Moskov. Mat. Obšc. 11 (1962), 199-242; English transl., Amer. Math. Soc. Transl. (2) 50 (1966), 5-58.

WASHINGTON UNIVERSITY AND

ST. Louis UNIVERSITY 